\title{
Quantification of recurrence risk based on number of adverse prognostic factors in women with stage I uterine endometrioid carcinoma
}

\author{
Andrew E. Cook ${ }^{1}$, (D) Ibrahim Aref ${ }^{1}$, (D) Charlotte Burmeister², (D) Miriana Hijaz ${ }^{3}$, (D) Mohamed A. Elshaikh1 \\ 1 Department of Radiation Oncology, Henry Ford Cancer Institute, Michigan, United States of America \\ 2Department of Public Health Sciences, Henry Ford Health System, Michigan, United States of America \\ 3Department of Women's Health, Division of Gynecologic Oncology, Henry Ford Hospital, Michigan, \\ United States of America
}

\section{Abstract}

Objective: The goal was to develop an updated model to predict the risk of recurrence, based on the number of adverse pathologic features in women with International Federation of Gynecology and Obstetrics stage I uterine endometrioid carcinoma, who did not undergo any adjuvant treatment.

Material and Methods: Women at a single center who underwent surgical staging without adjuvant therapy between January 1990 and December 2019 were included. Cox proportional hazards model was used to identify independent predictors of relapse free survival (RFS). Prognostic groups were then created based on the number of independent predictors of recurrence that were identified (0, 1 , or 2-3 risk factors). Overall survival (OS) and disease specific survival (DSS) were also calculated for each group.

Results: In total 1133 women were eligible for inclusion. Median follow-up was 84 months. Independent prognostic factors of recurrence included: age $\geq 60$; grade 2 or 3 differentiation; and presence of lymphovascular space invasion (LVSI). Due to the small number of patients with either 2 or 3 risk factors, these groups were combined into one (group 2/3). Isolated vaginal cuff recurrence was the most common site of recurrence in all study groups ( $2 \%, 7 \%$, and $17 \%$ for groups 0,1 , and $2 / 3$, respectively). Five-year RFS rates were $96 \%, 85 \%$, and $57 \%$ for groups 0 , 1 , and 2/3 (p<0.01), respectively. Five-year DSS rates were $99 \%, 96 \%$, and $85 \%$ and 5 -year OS rates were $94 \%, 85 \%$, and $62 \%$ (p<0.01), respectively.

Conclusion: We identified older age, high grade, and presence of LVSI as independent predictors of recurrence for women with stage I uterine endometrioid carcinoma. Using these prognostic factors, recurrence risk can be quantified for individual patients, and these factors can be used in deciding the appropriate adjuvant management course. (J Turk Ger Gynecol Assoc 2021; 22: 262-7)

Keywords: Endometrial adenocarcinoma, recurrence, prognosis, survival

Received: 26 April, 2021 Accepted: 30 July, 2021

\section{Introduction}

Endometrial carcinoma (EC) remains the most commonly diagnosed gynecologic cancer in the United States (1). Most women present with early-stage disease, with excellent survival outcomes (2).

Hysterectomy is the standard of care for women with earlystage EC, resulting in most patients being cured. However, some groups of patients are at risk for cancer recurrence due to the presence of some pathologic risk factors. The Gynecologic Oncology Group-99 (GOG-99) study showed that women with some adverse pathologic features who did not undergo adjuvant treatment have a recurrence risk of $26 \%$ at 2 years. This trial solidified the classic definition of high-intermediate risk in stage I EC as follows: age $\geq 70$ with one risk factor (grade 2 or 3 differentiation, presence of lymphovascular space invasion 
(LVSI), or deep myometrial invasion); age $\geq 50$ with any two risk factors; and any age with all three risk factors (3). Women with only one of these risk factors experience lower recurrence rates of $5 \%$ at 5 years after adjuvant radiation therapy (RT) (4). The National Comprehensive Cancer Network lists observation, $\mathrm{RT}$, and chemotherapy as treatment options for patients with International Federation of Gynecology and Obstetrics (FIGO) stage I EC (5). Given the number of treatment options and their associated toxicities, determining recurrence risk for this group of patients is vital so that appropriate adjuvant treatments can be selected.

Outside of prospective trials, there have been several studies examining recurrence in solely early-stage patients with EC (621). However, some of these studies include a heterogenous group of women with non-endometrioid histologies, such as serous carcinoma, which are known to have a poor prognosis $(8,10,11,21,22)$. All of these studies also include women who received various adjuvant treatments (6-21). Adjuvant therapy is known to significantly impact recurrence rates $(3,23)$. Furthermore, some studies utilized nomograms, which can be cumbersome to use in daily clinical practice (7).

A simplified prognostic model for women with FIGO stage I EC was recently reported based on tumor grade, presence of LVSI, and percent myometrial invasion. While useful, the investigators reported that older age at diagnosis was not an independent risk factor for cancer recurrence (24).

In order to examine recurrence rates in women with FIGO stage I EC accurately, a population of women who did not undergo any adjuvant treatment is needed to answer this question. By including a larger study cohort with a longer follow-up, we sought to analyze independent predictors of recurrence in women with FIGO stage I uterine EC who did not receive any adjuvant therapies.

\section{Material and Methods}

After obtaining Henry Ford Institutional Review Board approval (approval number: IRB 4645), we searched our prospectively maintained database for women with 2009 FIGO stage I EC who underwent hysterectomy and no adjuvant therapy between January 1990 and December 2019. As this was a retrospective study, informed consent was waived. Exclusion criteria were the presence of synchronous malignancies and non-endometrioid histology. After surgery, patients underwent routine follow-up with surveillance testing performed as clinically indicated. Follow-up data, including timing of recurrence, was collected from patients' medical records when available. Patient demographics, surgical pathologic variables, and survival endpoints were collected. Baseline comorbidity burden (Charlson comorbidity score) was also collected for each patient immediately before hysterectomy.

\section{Statistical analysis}

The primary endpoint of the study was relapse free survival (RFS), which included both locoregional and distant recurrences. Univariate (UVA) and multivariate analyses (MVA) were first performed to determine independent predictors of RFS. Cox proportional hazards models were used to identify independent predictors of recurrence using a manual stepwise selection with an entry criterion of $\mathrm{p}<0.2$ and stay criteria of $\mathrm{p}<0.05$. Groups were then created based on the number of recurrence risk factors that were present. Then, for each of these groups, RFS, overall survival (OS), and disease specific survival (DSS) were determined using date of hysterectomy as the start date. Kaplan-Meier plots were generated for each of these outcomes. Nominal and numerical variables were analyzed using a chi-square test and Student's t-test, respectively. All analyses were performed in statistical software SAS version 9.4 (SAS Institute Inc., Cary, NC).

\section{Results}

After considering inclusion and exclusion criteria, a total of 1,133 patients were included with a median (range) follow-up of 86.8 (1-346) months. Table 1 summarizes baseline patient characteristics, pathologic findings, and recurrence patterns for the study cohort. The median age was 60 (26-93) years. There were 75 patients (6.6\%) with FIGO stage IB disease. Pelvic lymph node (LN) dissection was performed in 59\% (666 patients) with or without para-aortic lymphadenectomy. The median (range) number of LN examined was 2 (0-66). For some patients with adverse prognostic factors, it was not clear in the medical records why they did not receive the recommended adjuvant treatment. Patient refusal was the most commonly reported reason for not receiving adjuvant treatment. There was a total of 71 women (6\%) who were diagnosed with cancer recurrence (pathologically, radiologically or both). The sites of their first relapse included isolated vaginal recurrence in 39/71 (54.9\%), isolated pelvic recurrence in four (5.6\%), vaginal and pelvic recurrence in nine (12.7\%), isolated paraaortic recurrence in two (2.8\%), and distant recurrence in 17 (23.9\%) patients.

For the entire cohort, predictors of worse 5-year RFS on UVA were age (as continuous and dichotomous variables), higher grade, higher stage, and presence of LVSI. On MVA predictors were older age $(\geq 60)$, grade $2 / 3$, and presence of LVSI (Table 2 ). These three independent predictors were then used to design the final recurrence model. A score of 0 was applied if the following factors were present: age $<60$, grade 1 , and lack of LVSI. A score of 1 was applied for the following risk factors: age $\geq 60$, grade $2 / 3$, and presence of LVSI.

Initially, four risks groups were created (group 0 with no risk factors, group 1 with one risk factor, group 2 with two risk 
factors, and group 3 with three risk factors). Due to the small number of patients in groups with 2 or 3 risk factors, these were combined into one group (group 2/3), creating a total of three risk groups.

There were 871 patients (77\%) in group 0, 220 patients (19\%) in group 1, and 42 patients (4\%) in group 2/3. Regarding baseline characteristics among the groups, there were significant differences noted in age, body mass index, characteristics of LN dissection, grade, lower uterine segment involvement, presence of LVSI, and stage (Table 1).

Five-year RFS rates for the three prognostic groups were 96\% [95\% confidence interval (CI) 95-97\%] for group 0, 85\% (95\% CI 78-90\%) for group 1, and 57\% (95\% CI 38-73\%) for group 2/3 $(\mathrm{p}<0.01)$ (Figure 1). Independent predictors for RFS included age $\geq 60$ years, higher tumor grade, and the presence of LVSI.

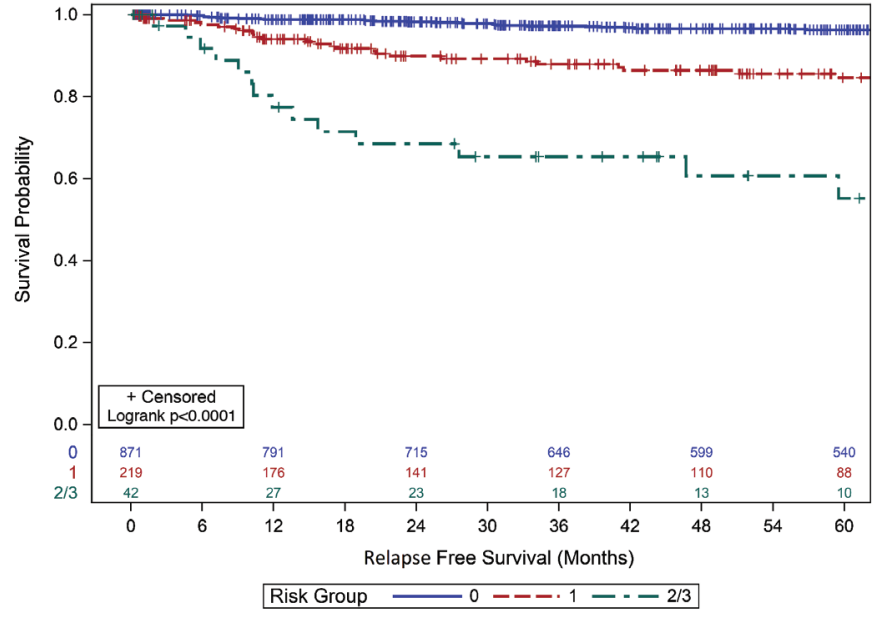

Figure 1. Kaplan-Meier plot comparing 5-year relapse free survival among risk groups

Table 1. Baseline patient characteristics across risk factor groups

\begin{tabular}{|c|c|c|c|c|}
\hline Variable & $\begin{array}{l}\text { Group 0 } \\
(n=871)\end{array}$ & $\begin{array}{l}\text { Group 1 } \\
(n=220)\end{array}$ & $\begin{array}{l}\text { Group 2/3 } \\
(n=42)\end{array}$ & $\mathbf{p}$ \\
\hline Age, median (range) & $59.0(26.0-91.0)$ & $62.0(30.0-92.0)$ & $73.5(34.0-93.0)$ & $<0.01$ \\
\hline Age $<60$ & $456(52 \%)$ & $89(40 \%)$ & $8(19 \%)$ & \multirow{2}{*}{$<0.01$} \\
\hline Age $>60$ & $415(48 \%)$ & $131(60 \%)$ & $34(81 \%)$ & \\
\hline Median (range) body mass index & $36.2(17.9-71.3)$ & $33.4(15.6-60.3)$ & $30.5(18.2-59.6)$ & $<0.01$ \\
\hline Median (range) Charlson Comorbidity score & $0(0-12)$ & $0(0-9)$ & $0(0-4)$ & 0.27 \\
\hline \multicolumn{4}{|l|}{ Race } & 0.39 \\
\hline White & $680(78 \%)$ & $159(72 \%)$ & $34(81 \%)$ & - \\
\hline African American & $160(18 \%)$ & $53(24 \%)$ & $7(17 \%)$ & - \\
\hline Other & $30(3 \%)$ & $8(4 \%)$ & $1(2 \%)$ & - \\
\hline FIGO stage IA & $871(100 \%)$ & $176(80 \%)$ & $11(26 \%)$ & $<0.01$ \\
\hline FIGO stage IB & $0(0 \%)$ & $44(20 \%)$ & $31(74 \%)$ & - \\
\hline \multicolumn{4}{|l|}{ Tumor grade } & $<0.01$ \\
\hline 1 & $871(100 \%)$ & $63(29 \%)$ & $6(14 \%)$ & - \\
\hline 2 & $0(0 \%)$ & $131(60 \%)$ & $21(50 \%)$ & - \\
\hline 3 & $0(0 \%)$ & $26(12 \%)$ & $15(36 \%)$ & - \\
\hline Lymphovascular space invasion & $0(0 \%)$ & $19(9 \%)$ & $28(67 \%)$ & $<0.01$ \\
\hline Lower uterine segment involvement & $83(10 \%)$ & $34(15 \%)$ & $10(24 \%)$ & $<0.01$ \\
\hline Positive peritoneal cytology & $12(1 \%)$ & $0(0 \%)$ & $1(2 \%)$ & 0.09 \\
\hline Lymph node dissection & $470(54 \%)$ & $163(74 \%)$ & $33(79 \%)$ & $<0.01$ \\
\hline Median number of nodes examined & $1.0(0.0-55.0)$ & $5.0(0.0-66.0)$ & $4.5(0.0-45.0)$ & $<0.01$ \\
\hline Median number of pelvic nodes examined & $1.0(0.0-41.0)$ & $5.0(0.0-44.0)$ & $3.5(0.0-36.0)$ & $<0.01$ \\
\hline Median number PA nodes examined & $0.0(0.0-32.0)$ & $0.0(0.0-25.0)$ & $0.0(0.0-17.0)$ & $<0.01$ \\
\hline Tumor recurrence & $28(3 \%)$ & $29(13 \%)$ & $14(33 \%)$ & $<0.01$ \\
\hline \multicolumn{5}{|l|}{ Site of first tumor recurrence } \\
\hline Isolated vaginal recurrence & $17(2 \%)$ & $15(7 \%)$ & $7(17 \%)$ & 0.23 \\
\hline Pelvic only recurrence & $1(0 \%)$ & $3(1 \%)$ & $0(0 \%)$ & 0.65 \\
\hline Vaginal and pelvic recurrence & $2(0 \%)$ & $4(2 \%)$ & $3(7 \%)$ & 0.35 \\
\hline PA only recurrence & $0(0 \%)$ & $2(1 \%)$ & $0(0 \%)$ & 0.28 \\
\hline Distant recurrence & $8(2 \%)$ & $5(2 \%)$ & $4(9 \%)$ & 0.67 \\
\hline
\end{tabular}


Five-year DSS rates for the three groups were 99\% (95\% CI 98$99 \%$ ) for group 0, 96\% (95\% CI 91-98\%) for group 1, and 85\% (95\% CI 66-93\%) for group 2/3 ( $<<0.01)$ (Figure 2). Independent significant predictors for DSS included age $\geq 60$ years and deep myometrial invasion.

Five-year OS for group 0 was 94\% (95\% CI 93-96\%), 85\% for group 1 (95\% CI 78-89\%), and 62\% (95\% CI 44-76\%) for group 2/3 $(\mathrm{p}<0.01)$ (Figure 3$)$. The independent predictors for OS were age $\geq 60$, grade $2 / 3$ differentiation, deep myometrial invasion, and high comorbidity burden.

Table 2 summarizes the results of MVA for the different survival endpoints including hazard ratios.

\section{Discussion}

Although prior studies have aimed at creating a method to predict recurrence for early-stage EC, a simplified model is needed that is based on women who were observed following surgery. Given the lack of data to answer this question, some investigators sought to develop a simplified risk stratification method for women with FIGO stage I EC with endometrioid histology who did not undergo any adjuvant therapy. The authors were able to include 976 patients and identified tumor

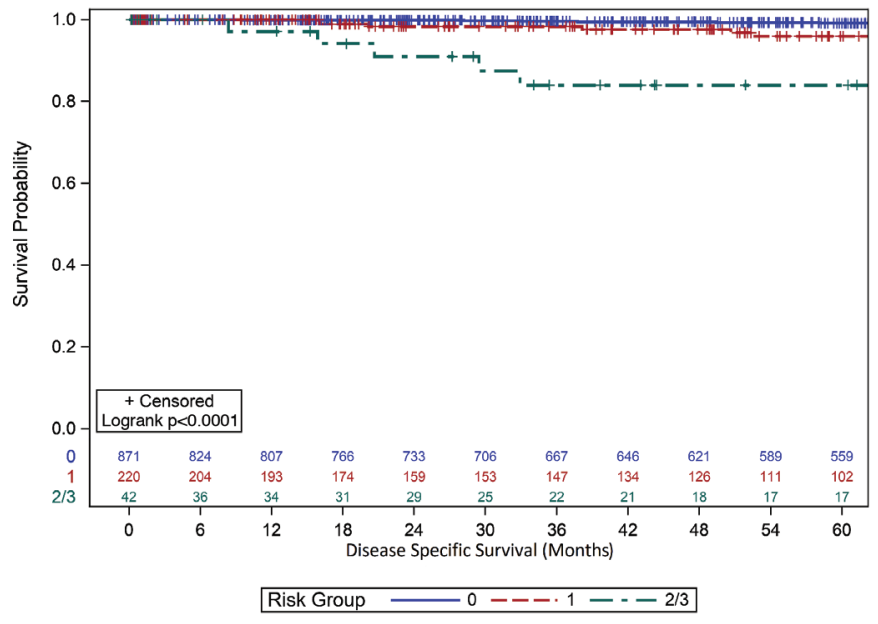

Figure 2. Kaplan-Meier plot comparing 5-year disease specific survival among risk groups grade 2/3, presence of LVSI, and stage IB as independent risk factors of recurrence, and three risk groups were established with 0,1 , or $2 / 3$ risk factors (24). The current study further builds on these results, using a larger patient cohort and, importantly, longer follow-up.

We were able to determine that independent predictors of 5-year RFS were older age, high tumor grade, and presence of LVSI. Utilizing these predictors, we created three separate risk groups based on the number of factors present: 0,1 , and $2 / 3$. The RFS survival endpoints for groups 0,1 , and $2 / 3$ were $96 \%, 85 \%$, and $57 \%$. This model provides a more individualized approach to determine patients' recurrence risk based on the number of risk factors.

Traditionally, patients with early-stage EC were grouped into low-risk, intermediate-risk, high-intermediate risk, and high risk $(3,23,25)$. These risk stratifications assume that all patients grouped into a specific category have the same risk of recurrence. This assumption is likely inaccurate given the definitions for each group are broad, leading to a heterogeneous cohort.

Also of note, our study confirms that the predominant pattern of first recurrence in women with early-stage EC is in the vagina. Overall, 39 patients (4\%) experienced vaginal relapse across

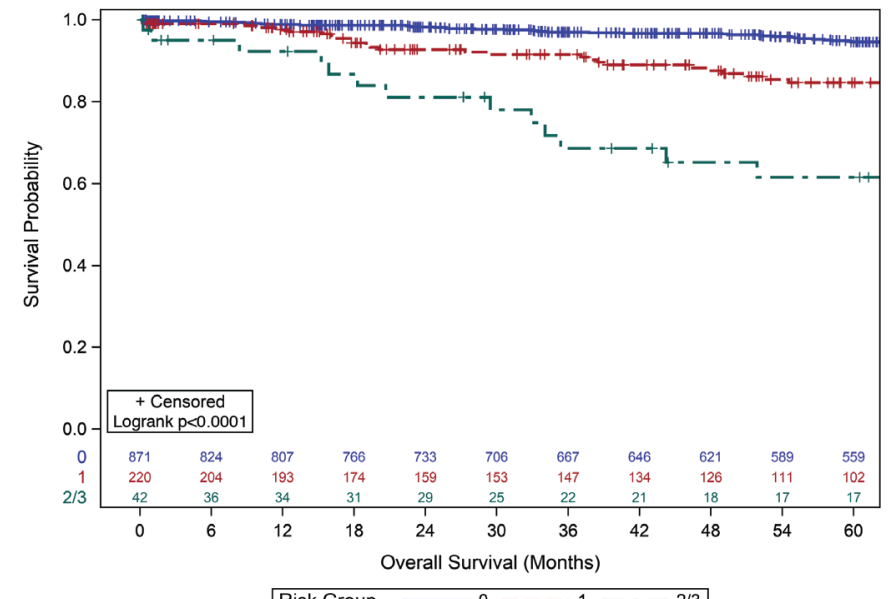

Figure 3. Kaplan-Meier plot comparing 5-year overall survival among risk groups

Table 2. Results of multivariate analyses of survival endpoints for the study cohort

\begin{tabular}{|c|c|c|c|c|c|c|c|c|c|}
\hline \multirow[b]{2}{*}{ Variable } & \multicolumn{3}{|c|}{ Relapse free survival } & \multicolumn{3}{|c|}{ Disease specific survival } & \multicolumn{3}{|c|}{ Overall survival } \\
\hline & HR & $95 \%$ CI & $\mathbf{p}$ & HR & 95\% CI & $\mathbf{p}$ & HR & $95 \%$ CI & $\mathbf{p}$ \\
\hline Older age ( $>60$ vs <60) & 1.03 & $1.01-1.05$ & 0.01 & 1.07 & $1.02-1.12$ & $<0.01$ & 1.07 & $1.04-1.09$ & $<0.01$ \\
\hline Positive lymphovascular space invasion & 4.52 & $2.27-8.97$ & $<0.01$ & 2.12 & $0.54-8.34$ & 0.28 & 1.72 & $0.83-3.60$ & 0.15 \\
\hline FIGO stage IB vs IA & 1.44 & $0.69-3.01$ & 0.33 & 3.87 & $1.19-12.57$ & 0.02 & 2.20 & $1.17-4.14$ & 0.01 \\
\hline
\end{tabular}


all three risk groups. This finding is in agreement with a large prospective trial (23). This recurrence pattern helps to inform appropriate adjuvant treatment options.

The recurrence rates for our patient population are similar to those that have been identified in prospective studies. For patients with 0 risk factors, we found a 5 -year RFS of $96 \%$, which is similar to a study by Sorbe et al. (26) in which women who were deemed low-risk (FIGO stage IA-IB, endometrioid histology, and FIGO grade 1-2) underwent observation and were found to have a recurrence rate of $4.1 \%$ at 5 years. Our 5 -year OS of $94 \%$ was also similar to this cohort, which was found to be $96.1 \%$ (26).

For our group with one risk factor, a comparable cohort was studied in the observation arm of Post Operative Radiation Therapy in Endometrial Carcinoma-1 (PORTEC-1), which included women with either $<50 \%$ myometrial invasion and grade $2 / 3$ disease or $>50 \%$ myometrial invasion and grade 1-2 disease. Our 5-year RFS for group 1 was $85 \%$, and this study showed a 5 -year recurrence rate of $16 \%$. Additionally, our 5-year OS was $85 \%$, which was identical to the endpoint examined in this study (27).

Finally, our group with $2 / 3$ risk factors can be compared to patients in the observation arm studied in GOG-99 (inclusion criteria described earlier). Our 5-year RFS was 57\%, which was not as favorable compared to the 5 -year recurrence rate of close to $30 \%$ in this study. The 5 -year OS for our group of $57 \%$ is also notably lower than the OS of approximately $75 \%$ found in this study. One possible explanation for this discrepancy is that we were able to include only 42 patients in this group while the GOG-99 study observed 70 high-intermediate risk patients (3). Additionally, as our study is retrospective, selection bias may explain the higher recurrence and lower survival in our patient cohort.

Of note, a dissimilar aspect between our study and many of the previously mentioned prospective studies is that myometrial invasion/stage was not found to be a significant predictor for recurrence in our analysis. Depth of myometrial invasion is a well-known predictor for recurrence, as demonstrated in the GOG-33 analysis (28). A possible explanation for us not identifying deep myometrial invasion as a predictor was that only 75 patients $(6.6 \%)$ had FIGO stage IB disease. It is still likely that myometrial invasion predicts for recurrence and should be considered when determining treatment management.

\section{Study limitation}

Our study does have some other limitations. As noted above, this study is retrospective and prone to selection bias in terms of which treatment the patients received. Also, there were only 42 patients who were included in group $2 / 3$. This small number of patients does limit the power of statistical analyses on this group and the conclusions that can be drawn from this data.
In addition to utilizing these risk factors to determine recurrence risk, a promising future method to help in this effort uses molecular prognostication. The Cancer Genome Atlas identified four molecular subgroups within EC: POLE ultramutated, microsatellite instability hypermutated, copy-number low, and copy-number high (29). PORTEC-4 is an ongoing study determining if these molecular groups are prognostic and if they can guide adjuvant treatment decisions (30). The results of this trial are eagerly awaited and may be practice changing.

\section{Conclusion}

This simplified recurrence model for patients with FIGO stage I EC includes three traditional independent predictors of RFS: older age, high tumor grade, and presence of LVSI. Given that risk groups defined by historical studies for this patient population are very heterogenous, this risk-scoring system can be applied to individual patients and is easy to utilize in daily clinical practice.

Ethics Committee Approval: The study was approved by Henry Ford Institutional Review Board (approval number: IRB 4645).

Informed Consent: As this was a retrospective study, informed consent was waived.

Peer-review: Externally peer-reviewed.

Author Contributions: Surgical and Medical Practices: A.E.C., I.A., M.H., M.A.E.; Concept: I.A., M.H., M.A.E.; Design: I.A., M.H., M.A.E.; Data Collection or Processing: A.E.C., C.B.; Analysis or Interpretation: A.E.C., I.A., C.B., M.H., M.A.E.; Literature Search: A.E.C., M.A.E.; Writing: A.E.C., M.A.E.

Conflict of Interest: No conflict of interest is declared by the authors.

Financial Disclosure: The authors declared that this study received no financial support.

\section{References}

1. Siegel RL, Miller KD, Jemal A. Cancer statistics, 2020. CA Cancer J Clin 2020; 70: 7-30.

2. Sheikh MA, Althouse AD, Freese KE, Soisson S, Edwards RP, Welburn S, et al. USA endometrial cancer projections to 2030: should we be concerned? Future Oncol 2014; 10: 2561-8.

3. Keys HM, Roberts JA, Brunetto VL, Zaino RJ, Spirtos NM, Bloss JD, et al.; Gynecologic Oncology Group. A phase III trial of surgery with or without adjunctive external pelvic radiation therapy in intermediate risk endometrial adenocarcinoma: a Gynecologic Oncology Group study. Gynecol Oncol 2004; 92: 744-51.

4. Sorbe B, Horvath G, Andersson H, Boman K, Lundgren C, Pettersson B. External pelvic and vaginal irradiation versus vaginal irradiation alone as postoperative therapy in medium-risk endometrial 
carcinoma--a prospective randomized study. Int J Radiat Oncol Biol Phys 2012; 82: 1249-55.

5. National Comprehensive Cancer Network. Uterine Neoplasms (Version 1.2021) https://www.nccn.org/professionals/physician gls/pdf/uterine.pdf

6. Francis SR, Ager BJ, Do OA, Huang YJ, Soisson AP, Dodson MK, et al. Recurrent early stage endometrial cancer: Patterns of recurrence and results of salvage therapy. Gynecol Oncol 2019; 154: 38-44.

7. Creutzberg CL, van Stiphout RG, Nout RA, Lutgens LC, JürgenliemkSchulz IM, Jobsen JJ, et al. Nomograms for prediction of outcome with or without adjuvant radiation therapy for patients with endometrial cancer: a pooled analysis of PORTEC-1 and PORTEC-2 trials. Int J Radiat Oncol Biol Phys 2015; 91: 530-9.

8. Helpman L, Perri T, Lavee N, Hag-Yahia N, Chariski HA, Kalfon S, et al. Impact of adjuvant treatment on outcome in high-risk earlystage endometrial cancer: a retrospective three-center study. Int $\mathrm{J}$ Gynecol Cancer 2019; 29: 133-9.

9. Yilmaz E, Gurocak S, Melekoglu R, Koleli I, Faydali S, Temelli O, et al. The Effect of Prognostic Factors and Adjuvant Radiotherapy on Survival in Patients with High-Grade Early-Stage Endometrial Cancer: A Retrospective Clinical Study. Med Sci Monit 2019; 25: 2811-8.

10. Jeppesen MM, Jensen PT, Gilså Hansen D, Iachina M, Mogensen $O$. The nature of early-stage endometrial cancer recurrence-A national cohort study. Eur J Cancer 2016; 69: 51-60.

11. Ly D, Rowley BD, Dodson MK, Soisson PA, Jolles CJ, Gaffney DK, et al. Adjuvant radiation in early stage, unfavorable histology endometrial carcinoma is associated with improved local control and survival. Gynecol Oncol 2014; 133: 250-5.

12. Reynaers EA, Jutzi L, Ezendam NP, Kwon JS, Pijnenborg JM. Improved outcome of high-grade, early 1-stage endometrioid endometrial carcinoma with adjuvant chemotherapy and radiotherapy: comparison of 2 treatment strategies. Int J Gynecol Cancer 2017; 27: 467-72.

13. Jin M, Hou X, Sun X, Zhang Y, Hu K, Zhang F. Impact of different adjuvant radiotherapy modalities on women with early-stage intermediate- to high-risk endometrial cancer. Int J Gynecol Cancer 2019; 29: 1264-70.

14. Cusano E, Myers V, Samant R, Sudai T, Keller A, Le T, et al. Prognostic Significance of Lymphovascular Space Invasion in the Absence of Lymph Node Metastases in Early-Stage Endometrial Cancer. Int J Gynecol Cancer 2018; 28: 890-4.

15. Laliscia C, Cosio S, Morganti R, Mazzotti V, Fabrini MG, Paiar F, et al. Patterns of Failures and Clinical Outcome of Patients with EarlyStage, High-Risk, Node-Negative Endometrial Cancer Treated with Surgery Followed by Adjuvant Platinum-Based Chemotherapy and Vaginal Brachytherapy. Oncology 2019; 96: 235-41.

16. Weinberg LE, Kunos CA, Zanotti KM. Lymphovascular space invasion (LVSI) is an isolated poor prognostic factor for recurrence and survival among women with intermediate- to high-risk earlystage endometrioid endometrial cancer. Int J Gynecol Cancer 2013; 23: 1438-45.

17. Hochreiter A, Kelly JR, Young MR, Litkouhi B, Black JD, Stromberger $\mathrm{C}$, et al. Outcomes and relapse patterns of stage IB grade 2 or 3 endometrial cancer treated with adjuvant vaginal brachytherapy. Int J Gynecol Cancer 2020; 30: 48-55.

18. Lan C, Huang X, Huang Q, Wang Y, Gu H, Li Y, et al. Should the optimal adjuvant treatment for patients with early-stage endometrial cancer with high-Intermediate risk factors depend on tumor grade? Int J Gynecol Cancer 2015; 25: 1445-52.

19. Nofech-Mozes S, Ackerman I, Ghorab Z, Ismiil N, Thomas G, Covens A, et al. Lymphovascular invasion is a significant predictor for distant recurrence in patients with early-stage endometrial endometrioid adenocarcinoma. Am J Clin Pathol 2008; 129: 912-7.

20. van der Putten LJ, Geels YP, Ezendam NP, van der Putten HW, Snijders MP, van de Poll-Franse LV, et al. Lymphovascular space invasion and the treatment of stage I endometrioid endometrial cancer. Int J Gynecol Cancer 2015; 25: 75-80.

21. Canlorbe G, Bendifallah S, Laas E, Raimond E, Graesslin O, Hudry $\mathrm{D}$, et al. Tumor size, an additional prognostic factor to include in low-risk endometrial cancer: results of a french multicenter study. Ann Surg Oncol 2016; 23: 171-7.

22. de Boer SM, Powell ME, Mileshkin L, Katsaros D, Bessette P, HaieMeder C, et al; PORTEC Study Group. Adjuvant chemoradiotherapy versus radiotherapy alone in women with high-risk endometrial cancer (PORTEC-3): patterns of recurrence and post-hoc survival analysis of a randomised phase 3 trial. Lancet Oncol 2019; 20: 127385.

23. Creutzberg CL, Nout RA, Lybeert ML, Wárlám-Rodenhuis CC, Jobsen JJ, Mens JW, et al; PORTEC Study Group. Fifteen-year radiotherapy outcomes of the randomized PORTEC-1 trial for endometrial carcinoma. Int J Radiat Oncol Biol Phys 2011; 81: e6318.

24. Elshaikh MA, Modh A, Sakr S, Shrestha R, Burmeister C, Ali-Fehmi $\mathrm{R}$, et al. A simplified risk stratification method for women with stage 1 endometrial carcinoma. Am J Clin Oncol 2019; 42: 131-7.

25. Colombo N, Creutzberg C, Amant F, Bosse T, González-Martín A, Ledermann J, et al; ESMO-ESGO-ESTRO Endometrial Consensus Conference Working Group. ESMO-ESGO-ESTRO Consensus Conference on Endometrial Cancer: diagnosis, treatment and follow-up. Ann Oncol 2016; 27: 16-41.

26. Sorbe B, Nordström B, Mäenpää J, Kuhelj J, Kuhelj D, Okkan S, et al. Intravaginal brachytherapy in FIGO stage I low-risk endometrial cancer: a controlled randomized study. Int J Gynecol Cancer 2009; 19: 873-8.

27. Creutzberg CL, van Putten WL, Koper PC, Lybeert ML, Jobsen JJ, Wárlám-Rodenhuis $\mathrm{CC}$, et al. Surgery and postoperative radiotherapy versus surgery alone for patients with stage-1 endometrial carcinoma: multicentre randomised trial. PORTEC Study Group. Post Operative Radiation Therapy in Endometrial Carcinoma. Lancet 2000; 355: 1404-11.

28. Morrow CP, Bundy BN, Kurman RJ, Creasman WT, Heller P, Homesley HD, et al. Relationship between surgical-pathological risk factors and outcome in clinical stage I and II carcinoma of the endometrium: a Gynecologic Oncology Group study. Gynecol Oncol 1991; 40: 55-65.

29. Cancer Genome Atlas Research Network, Kandoth C, Schultz $\mathrm{N}$, Cherniack AD, Akbani R, Liu Y, et al. Integrated genomic characterization of endometrial carcinoma. Nature 2013; 497: 6773.

30. van den Heerik ASVM, Horeweg N, Nout RA, Lutgens LCHW, van der Steen-Banasik EM, Westerveld GH, et al. PORTEC-4a: international randomized trial of molecular profile-based adjuvant treatment for women with high-intermediate risk endometrial cancer. Int $\mathrm{J}$ Gynecol Cancer 2020; 30: 2002-7. 